\title{
Preliminary Outcome of Case Series of the Management of Unilateral and Bilateral Craniomandibular Ankylosis in Kenya
}

\section{-An Ongoing Prospective Study}

\author{
F. M. Butt ${ }^{*}$, S. W. Guthua ${ }^{2}$, E. M. Kegereki ${ }^{3}$ \\ ${ }^{1}$ Department of Human Anatomy and Oral \& Maxillofacial Surgery, University of Nairobi, Nairobi, Kenya \\ ${ }^{2}$ Department of Oral \& Maxillofacial Surgery, University of Nairobi, Nairobi, Kenya \\ ${ }^{3}$ Department of Periodontology/Community and Preventive Dentistry, University of Nairobi, Nairobi, Kenya \\ Email: ${ }^{\text {fawziamaxfax@gmail.com }}$
}

Received 24 July 2015; accepted 22 September 2015; published 28 September 2015

Copyright (C) 2015 by authors and Scientific Research Publishing Inc.

This work is licensed under the Creative Commons Attribution International License (CC BY).

http://creativecommons.org/licenses/by/4.0/

\section{(c) (i) Open Access}

\begin{abstract}
Temporomandibularmandibular ankylosis also known as the craniomandibular joint (CMJ) ankylosis is a Greek terminology that means "stiff joint". It is most commonly associated with trauma and to a lesser extent middle ear infections. The condition is debilitating with regard to nutrition, mantainance of oral hygiene, growth and the overall psychology state of the patient. In this paper we present preliminary results of an ongoing study to assess the clinical parameters, for example, maximum inter incisal opening (MIO) and chin deviation (CD) of patients with CMJ ankylosis receiving early surgical intervention.
\end{abstract}

\section{Keywords}

Temporo/Craniomandibular Joint Ankylosis, Trismus, Costochondral Graft

\section{Introduction}

Temporomandibularmandibular also termed as the craniomandibular joint (CMJ) ankylosis is a Greek terminology that means "stiff joint". It is defined as "the inability to open the mouth due to intracapsular or extracapsular pathology, either, fibrous or bony union between the head of the condyle and the glenoid fossa, or in the case of extracapsular, between the coronoid process and the zygomatic arch” [1]. It is most commonly associated with

${ }^{*}$ Corresponding author.

How to cite this paper: Butt, F.M., Guthua, S.W. and Kegereki, E.M. (2015) Preliminary Outcome of Case Series of the Management of Unilateral and Bilateral Craniomandibular Ankylosis in Kenya. Open Journal of Stomatology, 5, $227-233$. http://dx.doi.org/10.4236/ojst.2015.59028 
trauma (13\% to $100 \%)$, local (middle ear infections) or systemic infection ( $0 \%$ to $53 \%$ ), or systemic disease, such as ankylosing spondylitis, rheumatoid arthritis, or psoriasis [2] [3]. Trauma can result in an intra-articular haematoma leading to fibrosis, excessive bone formation and ultimately hypomobility of the joint, especially in children [4]. Several authors have postulated that intra-articular haematoma alone may lead to ankylosis of the TMJ based on organisation and subsequent ossification of an intracapsular haematoma [5]-[9]. Oztan et al., however, disputed this theory, saying that trauma in the TMJ did not always progress to form bone [10]. Laskin on the other hand attributed the ankylosis to meniscal tear, which led to the fusion of the glenoid and condyle with hematoma formation [11]. Surprisingly, ankylosis still occurs in children with condylar head fractures where meniscus is intact, so the pathogenesis is indeed intriguing and still a partially resolved problem [5] [12] [13].

There are various classification of CMJ ankylosis based on the tissue involved: bony or fibrous, anatomical site: intracapsular or extracapsular and the number of joints affected: unilateral or bilateral [1]. The effects of ankylosis on patients are debilitating ranging from a compromised masticatory function, impairment of speech, to poor oral hygiene contributing to rampant caries and odontogenic infections. In addition, it also leads to interference in orofacial growth and development leading to facial asymmetry and micrognathia that may lead to airway embarrassment in case of life-threatening odontogenic infections such as, Ludwig's Angina [14]. All these have a detrimental effect on growth, nutritional and psychological status of the patient, and hence the need for surgical intervention. It is therefore imperative to plan the management with a multidisciplinary team consisting of a maxillofacial surgeon, anaesthesiologist, dentist, orthodontist, nutritionist, physiotherapist and psychologist. The surgical treatment of TMJ ankylosis may involve the 7-step protocol depending on the severity of the ankylosis, that is, Type I, II, III, or IV [12] [15]. Gap arthroplasty which is faster and less involving has been advocated, however, it has the disadvantage and drawback of re-ankylosis.

The aim of this study was to assess the effect of early intervention of TMJ ankylosis on the facial profile and growth of children attending the University of Nairobi Dental Teaching Hospital.

\section{Materials and Methods}

All patients with TMJ ankylosis who were either referred or self-referred to the University of Nairobi Dental Hospital Centre, Oral and Maxillofacial clinic, were included in the study between 2010 and 2013. The major presenting complaint was the inability to open the mouth, hence, causing difficulty in feeding and facial deformity. Possible causes of ankylosis, for example, trauma, and infection among others were recorded from the parents or the guardians' history. The patients were subjected to a comprehensive head, neck and oral examination. The maximum interincisal opening (MIO) and the extent of chin deviation (CD) from the midline were recorded for each patient for pre-, intra-, and postoperative reviews. Pre-operative intra oral examination was difficult due the limitation in mouth opening; however, the general oral hygiene was assessed. Photographs were taken to document the patient's facial profile pre-, intra- and post operatively during follow up. Plain radiographs (orthopantograms) in the majority of the cases, were used to evaluate the degree of ankylosis and CT Scans for those who were able to afford the cost of the investigation. Both OPG and coronal CT scan showed the typical mushroom shaped radio-opacity around the CMJ with obliteration of the space between the glenoid cavity and the condyle (Sawhney type IV) [12] (Figure 1).

All patients underwent blind nasotrachael intubation in theatre. The ankylosed CMJ was exposed through the preauricular incision. The surgeon followed a universally accepted 7-step protocol. This consisted of aggressive resection of the bony ankylotic mass to release the ankylosis, coronoidectomy on the affected side, coronoidotomy on the contralateral side and lining of the glenoid fossa with part of temporalis myofascial flap. Reconstruction of the condyle unit was achieved using a costochondral graft securing with rigid or semi-rigid fixation technique. The graft was harvested from either the 5 th or the 6th ribs where one graft was required, or from both in the case of bilateral ankylosis. Intraoperatively, all patients had an MIO of between 35 to $45 \mathrm{~mm}$ (Figure 2). A costochondral graft was used to maintain the vertical height of the ramus to re-establish function and improve the growth of the affected side of the mandible. Postoperatively, aggressive mouth opening exercises were began from the 1st post-operative day until discharge and the parents/guardians were counselled regarding their active role in adherence to aggressive home based physiotherapy.

The patients were discharged after 5 to 7 days, following an unremarkable recovery. The participants were subsequently reviewed in first two weeks, then in a month and every 3-monthly thereafter, to record the MIO, $\mathrm{CD}$, growth and secondary new traumatic or infective episode of otitis media. 


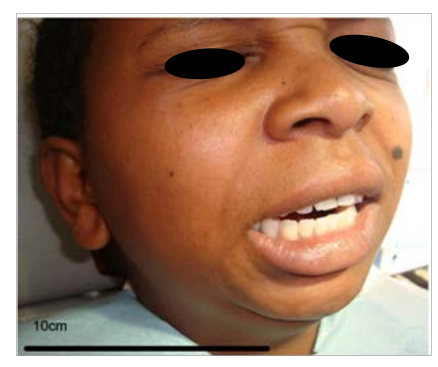

(a)

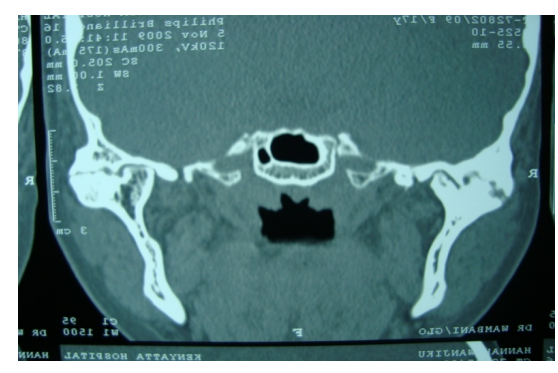

(b)

Figure 1. (a) PHOTO showing a patient with Bilateral TMJ ankylosis showing limited MIO. (b) CORONAL CT SCAN: Yellow arrows show the "Typical Mushroom” shaped ankylotic mass with almost complete obliteration of the joint space.

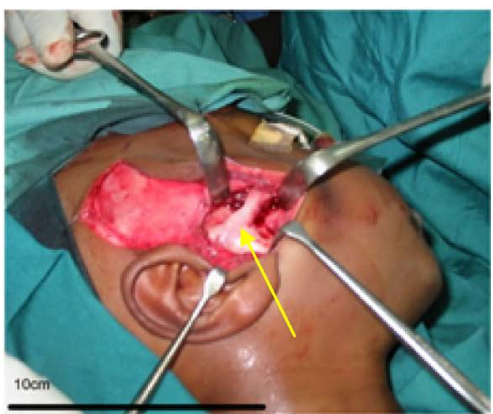

$\left(a_{i}\right)$

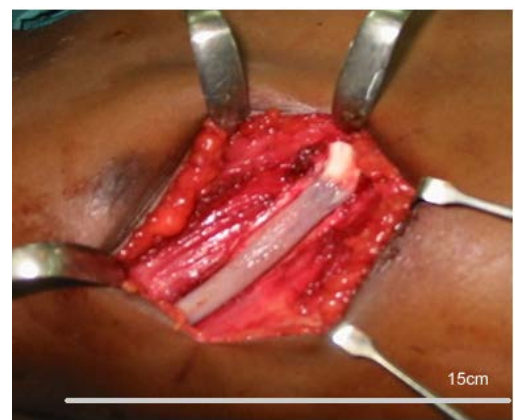

(b)

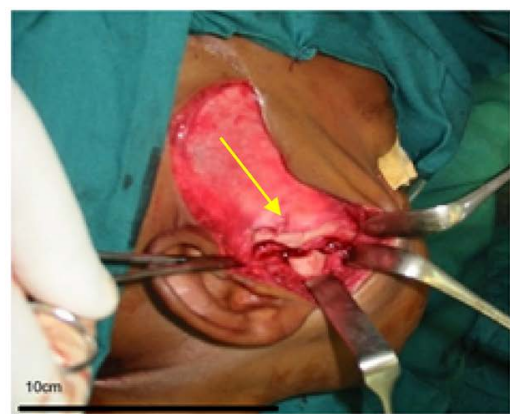

$\left(a_{\mathrm{ii}}\right)$

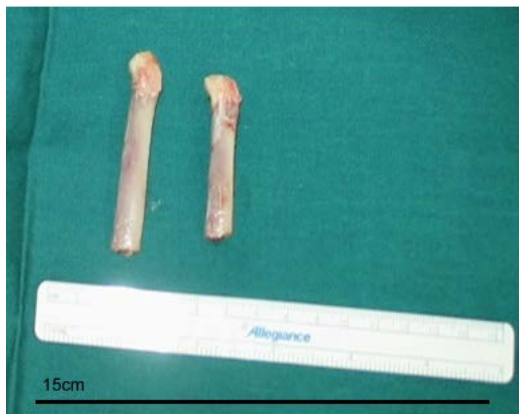

)

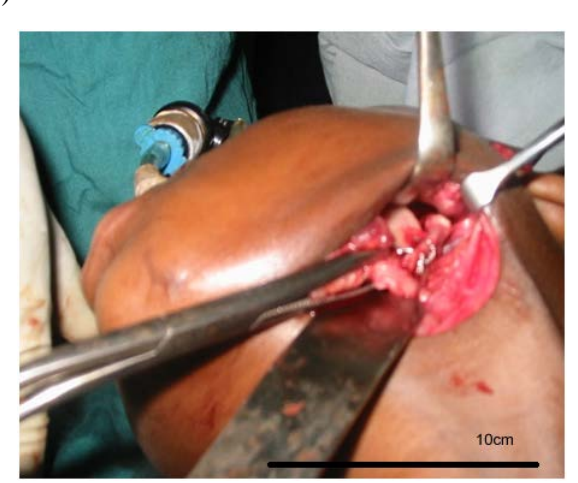

(c)

Figure 2. $\left(\mathrm{a}_{\mathrm{i}}\right)$ Intra-operative photos showing the preauricular incision used to expose the ankylotic mass with complete fusion of condyle and glenoid fossa (yellow arrow); $\left(\mathrm{a}_{\mathrm{ii}}\right)$ Arrow shows "Pseudo joint space" created after resection of the condylar head and ankylotic mass. (b) Harvesting: Costochondral grafts (same access) 5th and 6th rib. (c) Use of part of the temporomyofascial flap interposed between the glenoid fossa and reconstruction of the defect using the rib cartilage and the graft secured on to the decorticated ramus with $0.5 \mathrm{~mm}$ stainless steel soft wire (semi-rigid fixation). 


\section{Results}

There were twelve (12) patients with CMJ ankylosis. They were between 1 and 10 years old of age. All had presented to the facility between 2010 and 2013. All the patients reported a positive history of trauma affecting the mandiblea. Some of the base line characteristics are shown in Table 1 . The radiographs showed that all had the bony type of ankylosis. Estimation methods by bootstrapping were implemented with the various parameters estimated within a 95\% confidence interval. These parameters were estimated for the CD, Pre-op MIO, Intra-op MIO and the Post-op MIO are summarized in Table 2.

There were twice as many males to females (M:F = 2:1), eight (8) of them had the unilateral and four (4) had the bilateral types of ankylosis. The age range of the patients was between 1 to 10 years old with mean of 6.9 years old.

Preoperative assessment showed facial asymmetry with the CD ranging from about 0 to 17 mm towards ankylosed side in cases of unilateral ankylosis. For those with bilateral ankylosis, the mandible was retrognathic and hypoplastic giving the patient's a kind of "birds" facial profile, with the chin almost central and the MIO was between 0 to $5 \mathrm{~mm}$ (Figure 3). Majority of the patients had a preoperative MIO between from 0 to 8 millimetres (mm) but those with bilateral ankylosis had the least, from 0 to $5 \mathrm{~mm}$; the oral hygiene was poor. The average MIO achieved intraoperatively, for all patients in this group was $35 \mathrm{~mm}$. A comparison of the pre-, intra- and postoperative MIO values showed an improvement after surgery. The majority of the patients after surgery were able to maintain a MIO of between 40 - $45 \mathrm{~mm}$, with the exception of one who had a $10 \mathrm{~mm}$ decrease in mouth opening after 6 months of review (Figure 4).

The least MIO of 0 - $5 \mathrm{~mm}$ was noted in those patients with bilateral CMJ ankylosis. The mean estimated improvement after the surgery was $26 \mathrm{~mm}$, CI [21.6 - 30.3] increase of the MIO. A large effect size of $5.1 \mathrm{~mm}$, CI $[3.0,7.7]$ was also noted. The patients with unilateral ankyloses ended up with a bigger MIO (mean, $30.8 \mathrm{~mm}$,

Table 1. Baseline characteristics.

\begin{tabular}{|c|c|c|c|c|c|c|c|}
\hline Case & Age & Gender & $\begin{array}{c}\text { Type of } \\
\text { Ankylosis }\end{array}$ & $\begin{array}{l}\text { Preoperative Chin } \\
\text { Deviation (mm) }\end{array}$ & $\begin{array}{l}\text { Preoperative } \\
\text { MIO (mm) }\end{array}$ & $\begin{array}{l}\text { Intra Operative } \\
\text { MIO (mm) }\end{array}$ & $\begin{array}{c}\text { Post Operative } \\
\text { MIO (mm) }\end{array}$ \\
\hline 1 & 1 & $\mathrm{~F}$ & Unilateral & 17 & 4 & 44 & $40-42$ \\
\hline 2 & 8 & M & Bilateral & 0 & 0 & 30 & $23-27$ \\
\hline 3 & 7 & M & Unilateral & 10 & 5 & 34 & $20-24$ \\
\hline 4 & 4 & M & Unilateral & 3 & 1 & 30 & $25-28$ \\
\hline 5 & 4 & M & Bilateral & 0 & 4 & 35 & $30-35$ \\
\hline 6 & 8 & $\mathrm{~F}$ & Unilateral & 11 & 0 & 34 & $32-35$ \\
\hline 7 & 5 & $\mathrm{~F}$ & Unilateral & 5 & 7 & 37 & $30-36$ \\
\hline 8 & 3 & M & Unilateral & 0 & 5 & 30 & $26-30$ \\
\hline 9 & 6 & M & Unilateral & 13 & 8 & 40 & $40-42$ \\
\hline 10 & 8 & M & Bilateral & 0 & 3 & 37 & $29-35$ \\
\hline 11 & 9 & M & Bilateral & 15 & 5 & 33 & $30-31$ \\
\hline 12 & 10 & $\mathrm{~F}$ & Unilateral & 0 & 3 & 36 & $33-34$ \\
\hline
\end{tabular}

Table 2. This was the average post-op MIO measured in mm.

\begin{tabular}{|c|c|c|c|}
\hline & \multicolumn{3}{|c|}{ Type of Ankylosis } \\
\hline & Total & Bilateral & Unilateral \\
\hline \multicolumn{4}{|l|}{ Gender } \\
\hline Female & $4(33 \%)$ & $0(0 \%)$ & $4(100 \%)$ \\
\hline Male & $8(67 \%)$ & $4(50 \%)$ & $4(50 \%)$ \\
\hline Age & $6( \pm 3)$ & $7( \pm 2)$ & $6( \pm 3)$ \\
\hline Pre-op CD & $3.8( \pm 2.5)$ & $3.0( \pm 2.2)$ & $4.1( \pm 2.7)$ \\
\hline Pre-op MIO & $6.2( \pm 6.6)$ & $3.8( \pm 7.5)$ & $7.4( \pm 6.3)$ \\
\hline Intra-op MIO & $35.0( \pm 4.2)$ & $33.8( \pm 3.0)$ & $35.6( \pm 4.8)$ \\
\hline Post-op MIO ${ }^{\ddagger}$ & $29.8( \pm 6.1)$ & $28.0( \pm 3.4)$ & $30.8( \pm 7.1)$ \\
\hline
\end{tabular}

\footnotetext{
${ }^{\ddagger}$ This was the average post-op MIO measured in mm.
} 


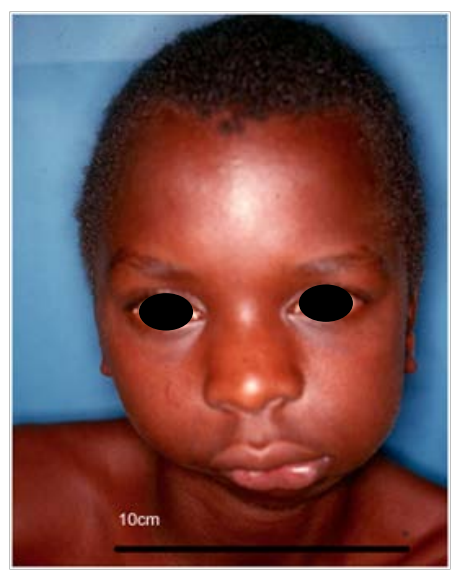

(a)

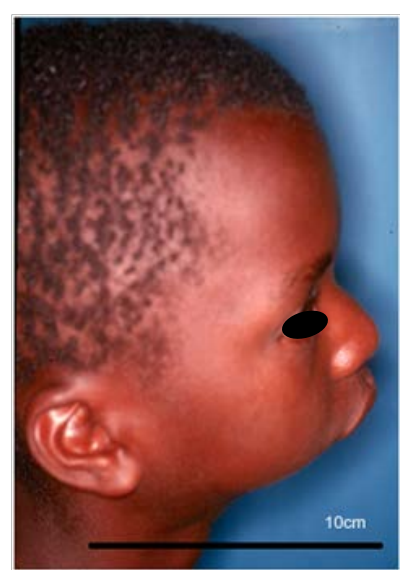

(b)

Figure 3. (a) Unilateral CMJ ankylosis showing the CD to the left side; (b) Side view shows "bird like" facial profile-mandibular hypoplasia (Retrognathia).

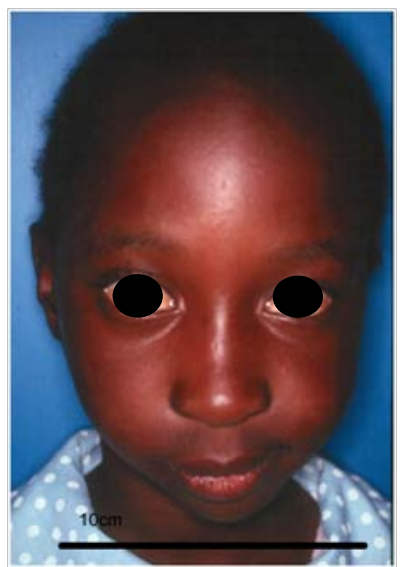

(a)

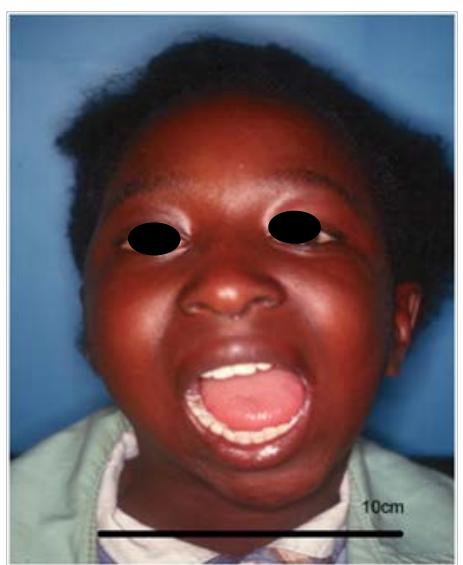

(b)

Figure 4. Unilateral TMJ ankylosis before (a) and 2 weeks after surgery with MIO of $45 \mathrm{~mm}$ (b). (a) Preoperative; (b) Postoperative.

CI [24.1, 37.3]), whereas the patients with the bilateral ankyloses ended up with a mean of $28.5 \mathrm{~mm}$ CI [19.7, 36.9]. The difference between these was estimated at $2.6 \mathrm{~mm}$, CI $[-7.8,13.9]$. The reduction in the MIO was found to be more varied amongst the patients with bilateral ankylosis, with an estimated standard deviation of $7 \mathrm{~mm}$ CI $[0.8,17.9]$ as compared to the more homogenous outcome amongst the patients with the unilateral ankyloses with a standard deviation of $8.5 \mathrm{~mm}$ CI $[3.9,14.9]$ (Table 3).

\section{Discussion}

Trauma and to a lesser extent infection are the two leading causes of CMJ ankylosis [16] [17]. All our patients had CMJ ankylosis due to trauma. There are serious consequences following TMJ ankylosis with regard to function and facial symmetry. Children have a greater tendency towards post traumatic ankylosis, mostly before the age of ten years old [18]. Laskin evaluated the factors which could contribute to the development of post traumatic CMJ ankylosis and summarised them into age of the patient, site and type of fracture, duration of immobilization and meniscal tear [11]. Considering the Moss functional matrix theory, it is imperative to note that restoration of jaw function will contribute to continued facial growth, hence, necessitating the need for early surgical intervention [19]. This approach does not only improve mastication and speech but also allows the patient to maintain oral hygiene which preserves the permanent dentition [4] [20]-[23].

Immediate surgical intervention following a diagnosis of intracapsular TMJ ankylosis will facilitate normal 
Table 3. Shows the average post-op MIO measured in mm.

\begin{tabular}{|c|c|c|c|c|}
\hline & Range & Mean & SD & Effect size \\
\hline \multicolumn{5}{|l|}{ ALL (n = 12) } \\
\hline Pre-op CD & $0-17$ & $6.0[1.4,6.0]$ & $7.4[4.2,11.2]$ & $0.9[0.2,1.6]$ \\
\hline Pre-op MIO & $0-8$ & $3.8[2.1,5.5]$ & $2.8[1.5,4.3]$ & $0.9[0.2,1.6]$ \\
\hline Intra-op MIO & $30-44$ & $34.9[32.1,37.7]$ & $4.5[2.4,7.1]$ & $8.2[4.3,12.5]$ \\
\hline Post-op MIO $^{\ddagger}$ & $20-40$ & $29.7[25.7,33.8]$ & $6.6[3.5,10.2]$ & $4.9[2.5,7.4]$ \\
\hline \multicolumn{5}{|l|}{ Unilateral $(n=8)$} \\
\hline Pre-op CD & $0-17$ & $7.3[1.5,13.3]$ & $7.7[3.6,13.3]$ & $1.1[0.1,2.1]$ \\
\hline Pre-op MIO & $0-8$ & $4.1[1.5,6.7]$ & $3.3[1.5,5.8]$ & $1.4[0.3,2.6]$ \\
\hline Intra-op MIO & $30-44$ & $35.5[31.2,40.0]$ & $5.7[2.6,9.9]$ & $7.0[2.8,11.4]$ \\
\hline Post-op MIO ${ }^{\ddagger}$ & $20-40$ & $30.7[23.9,37.1]$ & $8.5[3.9,14.8]$ & $4.0[1.6,6.6]$ \\
\hline \multicolumn{5}{|l|}{ Bilateral $(n=4)$} \\
\hline Pre-op CD & $0-15$ & $3.4[-15.7,22.1]$ & $14.9[0.0,39.6]$ & $0.3[-0.7,1.4]$ \\
\hline Pre-op MIO & $0-5$ & $3.0[-2.3,8.1]$ & $4.3[0.7,11.0]$ & $1.1[-0.5,2.8]$ \\
\hline Intra-op MIO & $30-37$ & $33.8[26.0,41.5]$ & $6.4[1.0,16.1]$ & $8.6[0.4,17.7]$ \\
\hline Post-op MIO $^{\ddagger}$ & $23-30$ & $27.9[19.5,36.1]$ & $6.8[0.5,17.4]$ & $6.9[0.2,14.7]$ \\
\hline \multicolumn{5}{|l|}{ Female $(n=4)$} \\
\hline Pre-op CD & $0-17$ & $9.6[-11.2,27.1]$ & $16.8[2.6,39.4]$ & $0.9[-0.4,2.3]$ \\
\hline Pre-op MIO & $0-7$ & $3.5[-4.0,10.5]$ & $5.7[1.0,14.9]$ & $0.9[-0.4,2.4]$ \\
\hline Intra-op MIO & $34-44$ & $37.6[27.0,48.0]$ & $8.4[1.0,21.9]$ & $6.8[0.5,14.2]$ \\
\hline Post-op MIO $^{\ddagger}$ & $30-40$ & $33.0[21.9,46.2]$ & $10.6[1.1,25.2]$ & $6.0[0.1,13.0]$ \\
\hline \multicolumn{5}{|l|}{ Male $(n=8)$} \\
\hline Pre-op CD & $0-13$ & $3.4[-2.4,9.2]$ & $6.7[2.5,13.1]$ & $0.6[-0.3,1.4]$ \\
\hline Pre-op MIO & $0-8$ & $3.7[0.9,6.5]$ & $3.4[1.4,6.2]$ & $1.3[0.1,2.4]$ \\
\hline Intra-op MIO & $30-40$ & $33.7[29.5,37.9]$ & $5.0[2.0,9.2]$ & $7.7[2.7,13.0]$ \\
\hline Post-op MIO ${ }^{\ddagger}$ & $20-40$ & $27.3[20.9,34.0]$ & $7.8[2.8,14.7]$ & $4.1[1.3,7.2]$ \\
\hline
\end{tabular}

physiology of mastication and facial growth based on functional matrix theory. The decision to resect the condyle instead of doing the gap arthroplasty was to avoid re-ankylosis. The costochondral graft infers growth potential influencing mandibular growth and remodelling which invariably will be transferred to the orofacial tissues. In our ongoing study, some of the preliminary unpublished data show a reduction in CD as long as the MIO was $25 \mathrm{~mm}$ and above and over a period of 1 year. The overall MIO was 5 - 10 times better immediately after surgery, although a small decrease was noticed during follow-up visits. This may attribute to a lapse in the home-based physiotherapy recommended at discharge as a result of fear of pain during jaw mobilization. The CD was noticed to be better although a longer follow up was needed for definitive conclusive recommendations. The oral hygiene was initially worse for patients with bilateral TMJ ankylosis due to the marked trismus and this improved post operatively for both the groups as they were able to brush their teeth

The use of costochondral grafts aims to re-establish a growth centre and restoration of mouth opening. The mandible increases in length as part of the facial growth through the influence of the functional matrix to provide means of adaptive modelling during this growth. In this study, the increase in MIO, markedly improved masticatory function from the first post operative day and subsequently, as reported by other authors [17]. As we continue to assess the $\mathrm{CD}$, and when the facial profile of these patients up to adolescent age, we intend to observe the effect of early intervention on correction of facial growth in children with CMJ ankylosis.

\section{Conclusion}

Our preliminary findings show that early surgical management of bony CMJ ankylosis can increase the MIO, improve the masticatory function and facilitate occlusal rehabilitation as long as early and active mobilization of the joint is implemented post operatively. The change in facial growth characteristics will be followed for a period of 5 to 10 years before the final outcome of this study is published. 


\section{References}

[1] Malik, N.A. (2002) Text Book of Oral \& Maxillofacial Surgery. Jaypee Brothers Medical Publishers (P) Ltd., New Delhi, 207-218.

[2] Gay-Escoda, C. and Arguero, M. (1994) La corrección quirúrgica de la anquilosis de la articulación temporomandibular. Descripción de siete casos. Avances en Odontoestomatología, 10, 74-85.

[3] Kaban, L., Pogrel, M.A. and Perrott, D.H. (1997) Complications in Oral and Maxillofacial Surgery. WB Saunders, Philadelphia.

[4] Kaban, L.B., Perrot, D.H. and Fischer, K. (1990) A Protocol for Management of Temporalmandibular Joint Ankylosis. Journal of Oral and Maxillofacial Surgery, 48, 1145-1151. http://dx.doi.org/10.1016/0278-2391(90)90529-B

[5] Ferreti, C., Bryant, R., Becker, P. and Lawrence, C. (2005) Temporomandibular Joint Morphology Following PostTraumatic Ankylosis in 26 Patients. International Journal of Oral and Maxillofacial Surgery, 34, 376-381. http://dx.doi.org/10.1016/j.ijom.2004.09.003

[6] Miller, G.A., Page Jr., H.L. and Griffith, C.R. (1975) Temporomandibular Joint Ankylosis: Review of Literature and Report of Two Cases of Bilateral Involvement. Journal of Oral Surgery, 33,792-803.

[7] El-Mofty, S. (1972) Ankylosis of the Temporomandibular Joint. Oral Surgery, Oral Medicine, Oral Pathology, 33, 650-660. http://dx.doi.org/10.1016/0030-4220(72)90375-1

[8] Meng, F.W., Zhao, J.L., Hu, K.J. and Liu, Y.P. (2009) A New Hypothesis of Mechanisms of Traumatic Ankylosis of Temporomandibular Joint. Medical Hypotheses, 73, 92-93. http://dx.doi.org/10.1016/j.mehy.2009.01.024

[9] Guven, O. (2000) A Clinical Study on Temporomandibular Joint Ankylosis. Auris Nasus Larynx, 27, 27-33. http://dx.doi.org/10.1016/S0385-8146(99)00045-0

[10] Oztan, H.Y., Ulusal, B.G. and Aytemiz, C. (2004) The Role of Trauma on Temporomandibular Joint Ankylosis and Mandibular Growth Retardations: An Experimental Study. Journal of Craniofacial Surgery, 15, 274-282. http://dx.doi.org/10.1097/00001665-200403000-00024

[11] Laskin, D.M. (1978) Role of the Meniscus in the Aetiology of Post-Traumatic TMJ Ankylosis in Children. International Journal of Oral Surgery, 7, 340-345. http://dx.doi.org/10.1016/S0300-9785(78)80106-9

[12] Sawhney, C.P. (1986) Bony Ankylosis of the Temporomandibular Joint: Follow-Up of 70 Patients Treated with Arthroplasty and Acrylicspacer Interposition. Plastic and Reconstructive Surgery, 77, 29-38. http://dx.doi.org/10.1097/00006534-198601000-00006

[13] Goss, A.N. and Bosanquet, A.G. (1990) The Arthroscopic Appearance of Acute Temporomandibular Joint Trauma. Journal of Oral and Maxillofacial Surgery, 48, 780-784. http://dx.doi.org/10.1016/0278-2391(90)90330-5

[14] Chidzonga, M.M. (1999) Temporal Mandibular Joint Ankylosis: Review of 32 Cases. British Journal of Oral and Maxillofacial Surgery, 37, 123-126. http://dx.doi.org/10.1054/bjom.1997.0089

[15] Kaban, L.B., Bouchard, C.B. and Troulis, M.J. (2009) A Protocol for Management of Temporomabibular Joint Ankylosis in Children. Journal of Oral and Maxillofacial Surgery, 67, 1966-1978. http://dx.doi.org/10.1016/j.joms.2009.03.071

[16] Akama, M.K., Guthua, S.W., Chindia, M.L. and Kahuho, S.K. (2009) Management of Bilateral TMJ Ankylosis in Children: A Case Report. The East African Medical Journal, 86, 45-48.

[17] Guthua, S.W., Maina, D.M. and Kahugu, M. (1995) Management of Post Traumatic TMJ Ankylosis in Children: A Case Report. The East African Medical Journal, 72, 471-475.

[18] Williams, J.L.I. (1994) Injuries of the Condyle and Coronoid Process. In: Rowe, N.L. and Williams, J.L.I., Eds., Maxillofacial Injuries, 2nd Edition, Churchill Livingstone, Edinburg, 417.

[19] Moss, M.L. (1979) Functional Cranial Analysis and the Functional Matrix. International Journal of Orthodontics, 17, 21-31.

[20] Sarma, U.C. and Dave, P.K. (1991) TMJ Ankylosis: An Indian Experience. Oral Surgery, Oral Medicine, Oral Pathology, 72, 660-664. http://dx.doi.org/10.1016/0030-4220(91)90006-X

[21] Posnick, J.C. and Goldstein, J.A. (1993) Surgical Management of TMJ Ankylosis in Paediatric Population. Plastic and Reconstructive Surgery, 91, 791-798. http://dx.doi.org/10.1097/00006534-199304001-00006

[22] Inkster, R.G. (1964) Osteology. In: Romanes, G.J., Ed., Cunningham’s Text Book of Anatomy, Oxford University Press, Oxford, 75-210.

[23] Lello, G.E. (1990) Surgical Correction of TMJ Ankylosis. Journal of Cranio-Maxillofacial Surgery, 18, 19-26. http://dx.doi.org/10.1016/S1010-5182(05)80599-2 